Journal of Engineering and Applied Sciences 14 (Special Issue 1): 3922-3926, 2019

ISSN: 1816-949X

(C) Medwell Journals, 2019

\title{
Mobile Location Estimation Based on Received Signal Strength using Circular Approach
}

\author{
${ }^{1}$ Surendra Shrestha, ${ }^{1}$ Reenu Mool and ${ }^{2}$ Chun Kwan Park \\ ${ }^{1}$ Department of Electronics and Computer Engineering, Pulchowk Campus, IoE, \\ Tribhuvan University, Nepal \\ ${ }^{2}$ Division of Navigation Information System, Mokpo National Maritime University, Mokpo, Korea
}

\begin{abstract}
Mobile location can be estimated based on Received Signal Strength (RSS). The RSS received from serving and neighboring Base Stations (BS) are used to estimate the mobile position which yields circles along which the mobile may lie. The circles intersect at the estimated mobile position. The RSS method is capable of being applied in existing systems without any hardware development. Experimental measurements of received power for the $900 \mathrm{MHz}$ of existing GSM system are made. The RSS is used to calculate the distance between transmitter and receiver based on known transmitter power and transmitter location and an appropriate path loss model. Four different path loss models were reviewed; Hata Model, Walfisch Ikegami (WI) Model, ECC Model and Lee Model. Walfisch Ikegami Model showed the best result in terms of Mean Absolute Error (MAE) and is used to estimate the position location of the Mobile Station (MS).
\end{abstract}

Key words: Localization, received signal strength, path loss model, base station, RSS method, GSM

\section{INTRODUCTION}

In wireless localization has become a key enabling technology in many emerging wireless applications and services. The performance of wireless localization techniques is easily affected by the signal propagation environment. Global Positioning System (GPS) is one of the most popular wireless localization techniques in outdoor environment. GPS can provide high accuracy, however, GPS service is not applicable in most of the indoor environments as the weak satellite signals are easily obstructed by the buildings. In addition, GPS receivers are generally expensive and consume high power which can limit its application. When GPS signal is not available cell towers can be used as reference nodes and the location dependent signal parameters like Received Signal Strength (RSS), Angle of Arrival (AOA), Time of Arrival (TOA) and Time Difference of Arrival (TDOA) can be measured from the wireless signals between the base stations and the mobile devices (Liu et al., 2007).

Propagation models in wireless communication have focused on predicting the average received signal strength at a given distance from the transmitter. A radio propagation model is a set of mathematical expressions, diagrams and algorithms used to represent the radio characteristics of a given environment. It characterizes the radio wave propagation as a function of frequency, distance and other conditions. As the path loss encountered along any radio link serves as the main factor for characterization of propagation for the link, radio propagation models typically focus on realization of the path loss (Khan et al., 2012).

Each individual telecommunication link has to encounter different terrain, path, obstructions and atmospheric conditions, hence, it is necessary to formulate the exact loss for all telecommunication systems in a single mathematical equation. Different models exist for different types of radio links under different conditions. The GSM system provides measurement reports about the signal attenuations from serving and neighboring base stations. In a real propagation environment, path loss and shadowing attenuate the signal power. Path loss including all of the possible loss elements associated with interactions between the propagating wave and any objects between the transmitting and receiving antennae increases with the signal travel distance. Numerous models have been

Corresponding Author: Chun Kwan Park, Division of Navigation Information System, Mokpo National Maritime University, Mokpo, Korea 
proposed. The models frequently used in wireless communication like Hata Model (Parmar and Nimavat, 2015) Walfisch-Ikegami (WI) Model (Iskander and Yun, 2002; ECC Model (Chebil et al., 2011) and Lee Model (Chebil et al., 2011) are considered to calculate the path loss.

\section{MATERIALS AND METHODS}

Mobile location estimation method: The Received Signal Strength (RSS) method is used to determine the position location of a target device. Depending on a path loss model, the distance between two nodes can be estimated by measuring the energy of the received signal at one end. The main goal of a position location system is to determine the coordinates of a target node. To estimate any unknown target node position, reference nodes must exist in the wireless coverage area with known geometrical coordination to map with measurements that are gathered to locate the target node position. The multilateration technique is used to measure the position of a target node in the RSS method. RSS method can be used in the systems without the need to install new hardware in both the network nodes and target nodes (Bhandare and Dixit, 2013) (Fig. 1 and 2).

If there are three points $\mathrm{N}_{1}-\mathrm{N}_{3}$ as shown in Fig. 1 and the distances from the reference points to object $\mathrm{T}$ are known, then the point of the intersection of the three circles is the supposed location of object $\mathrm{T}$. The mobile device is located by the intersection point of 3 circles. Each circle has a radius of the distance between the mobile device and the considered BS.

The equations for three intersecting circles with centers at the reference points and radii equal to distance from the Target $\mathrm{T}$ are:

$$
\begin{gathered}
\mathrm{d}_{1}^{2}=\mathrm{x}^{2}+\mathrm{y}^{2} \\
\mathrm{~d}_{2}^{2}=\left(\mathrm{x}-\mathrm{x}_{2}\right)^{2}+\mathrm{y}^{2} \\
\mathrm{~d}_{3}^{2}=\left(\mathrm{x}-\mathrm{x}_{3}\right)^{2}+\left(\mathrm{y}-\mathrm{y}_{3}\right)^{2}
\end{gathered}
$$

where, $d_{1}$ is line between reference point $\mathrm{N}_{1}$ and Target point $T, d_{2}$ is the line between reference point $N_{2}$ and $T, d_{3}$ is the line between reference point $\mathrm{N}_{3}$ and $\mathrm{T}$, the coordinates of the Target point $\mathrm{T}$ are $(\mathrm{x}, \mathrm{y})$ and the coordinates of reference point are $(0,0)$, the coordinates

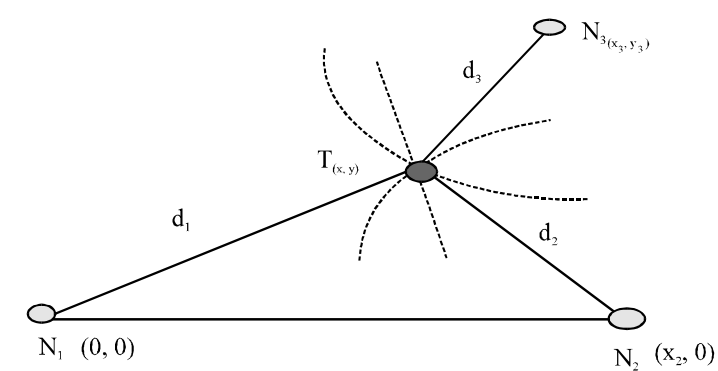

Fig. 1: Multilateration principle for position location of MS

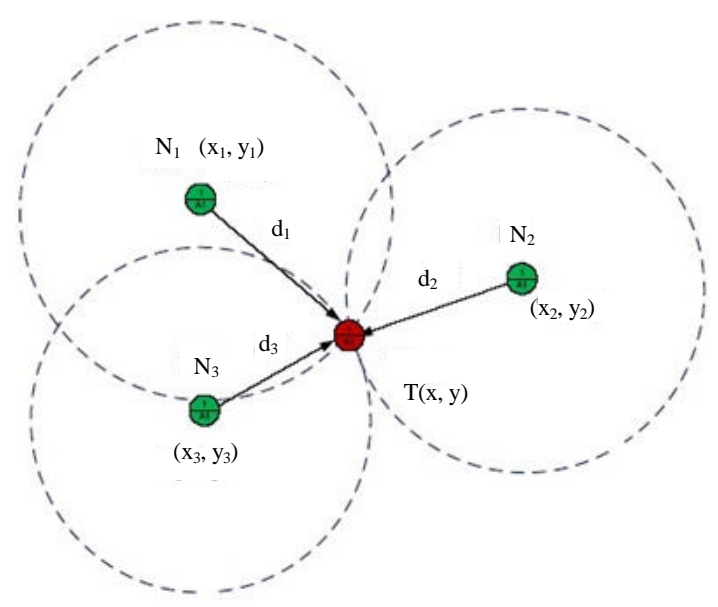

Fig. 2: RSS method for location estimation

of reference point $\mathrm{N}_{2}$ are $\left(\mathrm{x}_{2}, 0\right)$ and the coordinates of reference point $\mathrm{N}_{3}$ are $\left(\mathrm{x}_{3}, \mathrm{y}_{3}\right)$. Solving the equation gives the coordinates:

$$
\begin{gathered}
x=\frac{x_{2}^{2}+d_{1}^{2}-d_{2}^{2}}{2 x_{2}} \\
y=\frac{x_{3}^{2}+y_{3}^{2}+d_{1}^{2}-d_{3}^{2}-2 x_{3}}{2 y_{3}}
\end{gathered}
$$

The coordinates of the Target $\mathrm{T}$ can be estimated and the position they define is the only one where all three circles intersect (Bhandare and Dixit, 2013).

Each reference node is considered to be located at the center of the circle. The target node lies on the circumference of the circle. The distance between the center of the circle (reference node) and the target node is the radius of the circle. The intersection of these three circumferences is the location of the target node.

High accuracy can be obtained when using RSS method in short-range and LOS environment. This method 


\section{J. Eng. Applied Sci., 14 (Special Issue 1): 3922-3926, 2019}

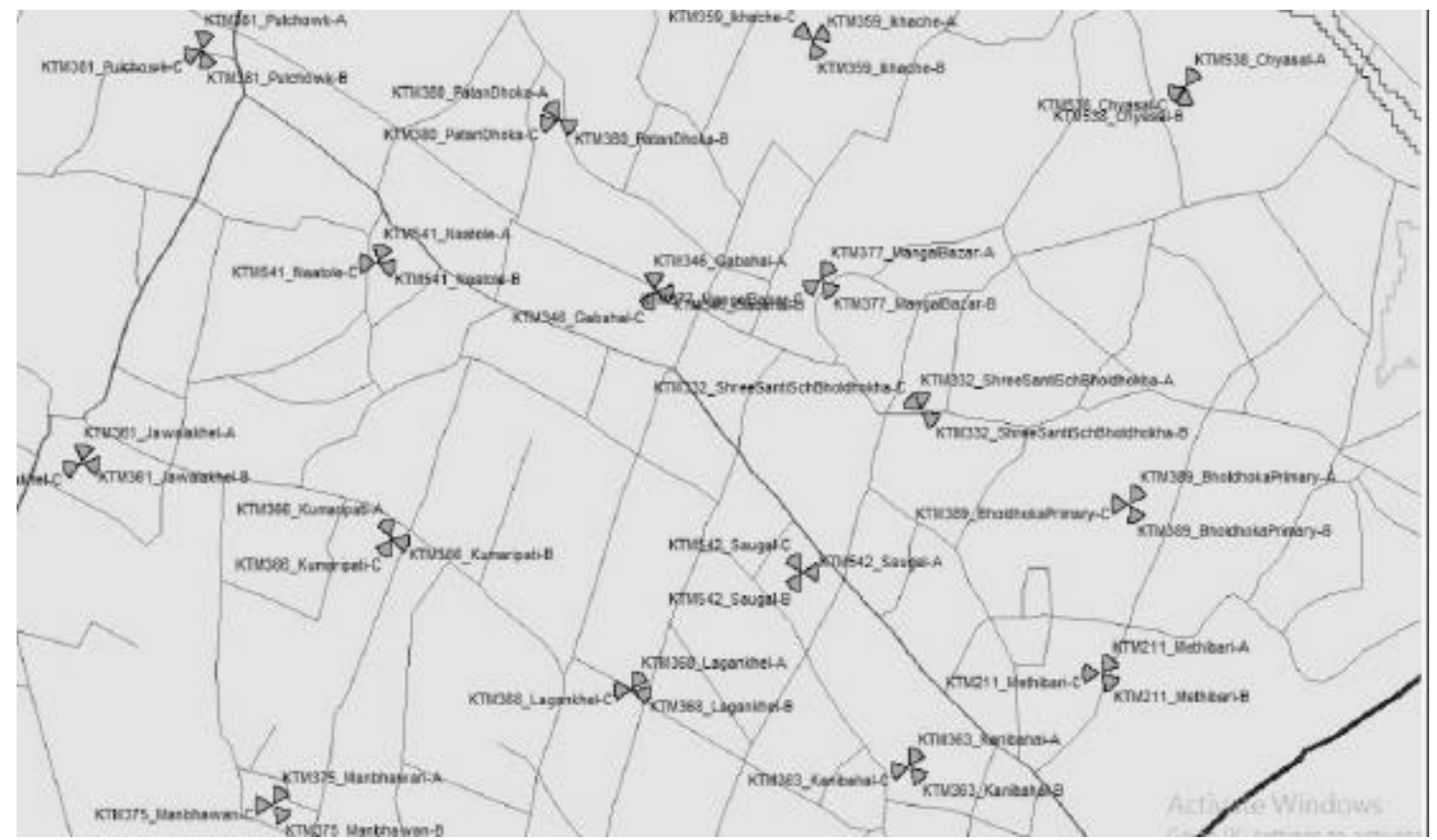

Fig. 3: Map of base stations taken into consideration (TEMS tool)

is easier and cheaper as compared to other methods that require installing new hardware in different nodes of the system. RSS method does not require time synchronization between nodes in the system.

For the real-time model around the geographic area, the place, Jawalakhel of Lalitpur District of Nepal has been chosen for the network. The place is located at $27^{\circ} 40^{\circ} 0^{\prime \prime} \mathrm{N}$, $85^{\circ} 19^{\prime} 0$ ' $\mathrm{E}$. The overlay region is around $(3 \times 3) \mathrm{km}^{2}$ consisting of nine Base Stations (BSs). The frequency of $900 \mathrm{MHz}$ was taken into consideration for the purpose. The height of the transmitter varies from 25-45 m whereas the building height varies from $10-16 \mathrm{~m}$. The area under consideration consists of both wide and narrow streets, hence, the road width varies from 6-14 $\mathrm{m}$. The height of the buildings varies from 10-16 m with the separation ranging from 1-3 m. The map of the area along with the BSs taken into consideration is shown in Fig. 3.

\section{RESULTS AND DISCUSSION}

The path loss calculated using Walfisch Ikegami Model varies linearly with the measured path loss throughout the distance between 100-700 m. The graph comparing the measured path loss with the path loss obtained from four different path loss models is shown in Fig. 4a-d.
Comparison is done in terms of Mean Absolute Error (MAE) (Willmott and Matsuura, 2005). It is observed that Walfisch-Ikegami Model has the lowest MAE of value $6.085 \times 10^{-3}$ for $900 \mathrm{MHz}$ as compared to the other models with MAE obtained highest from Lee Model with value $3.054 \times 10^{-1}$. Hence, WI Model was chosen to estimate the distance between the BS and MS.

The estimated distance obtained from WI Model is then used to locate the mobile position using a circular approach. The reference point receives the signal from 3-4 $\mathrm{BSs}$, however, only three BS which gave the minimum distances were taken into consideration. The circles are drawn using the tool MapInfo professional and the Graph tool. The center of the circle denotes the position of the BSs and the radius denotes the distance between the BS and MS. The three circles intersect exactly at the same point in the actual distance measurement as shown in Fig. 5a. However, in actual condition, it is observed that the three circles do not intersect at a common point due to multipath loss and shadowing effect. In such a case, the midpoint of the shortest distance between two circles is regarded as the point of intersection as given by Lin and Juang (2005). The mobile location is determined at the mean of the intersections which is the centroid of the intersections as shown in Fig. 5b.

Minimum error obtained using Walfisch Ikegami Model is around $22 \mathrm{~m}$ while the maximum error is around $159 \mathrm{~m}$. 
J. Eng. Applied Sci., 14 (Special Issue 1): 3922-3926, 2019
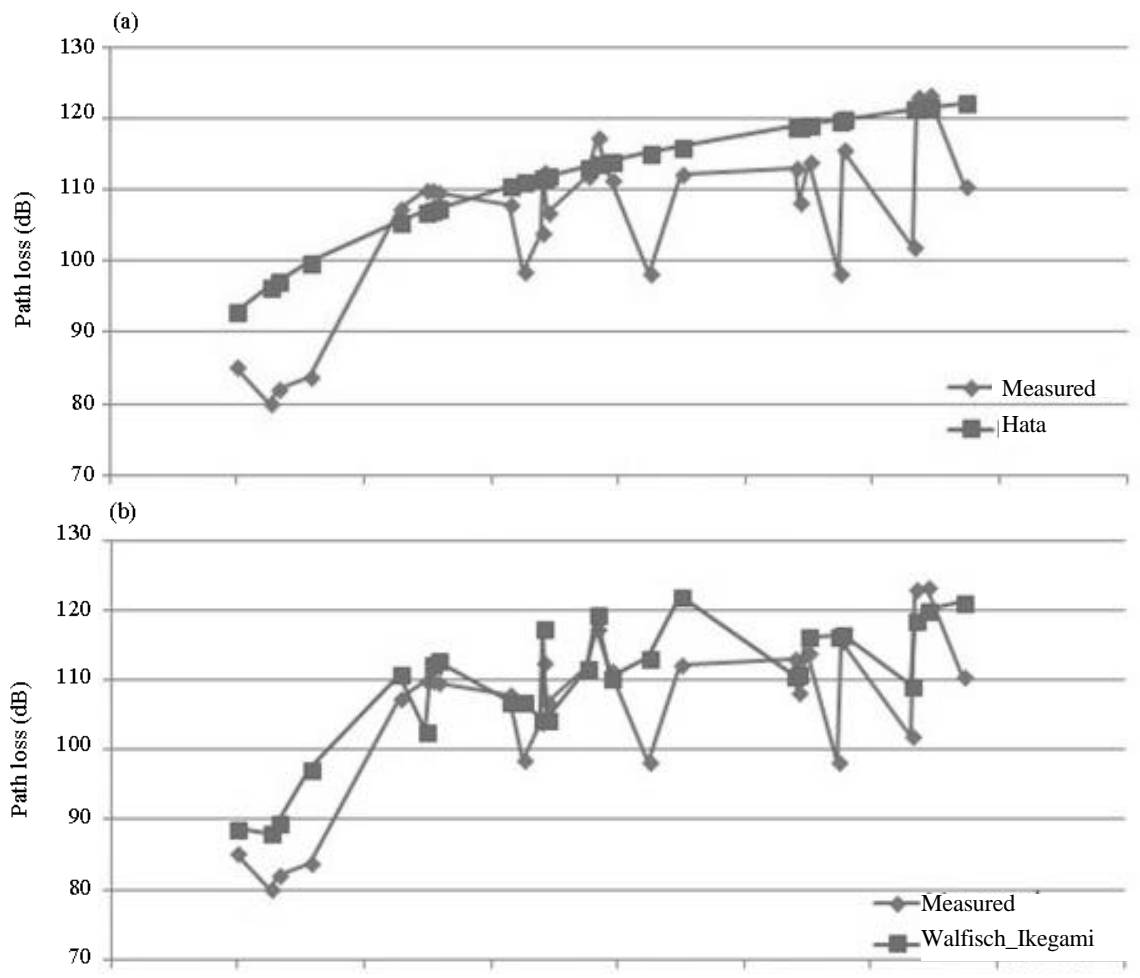

(c)
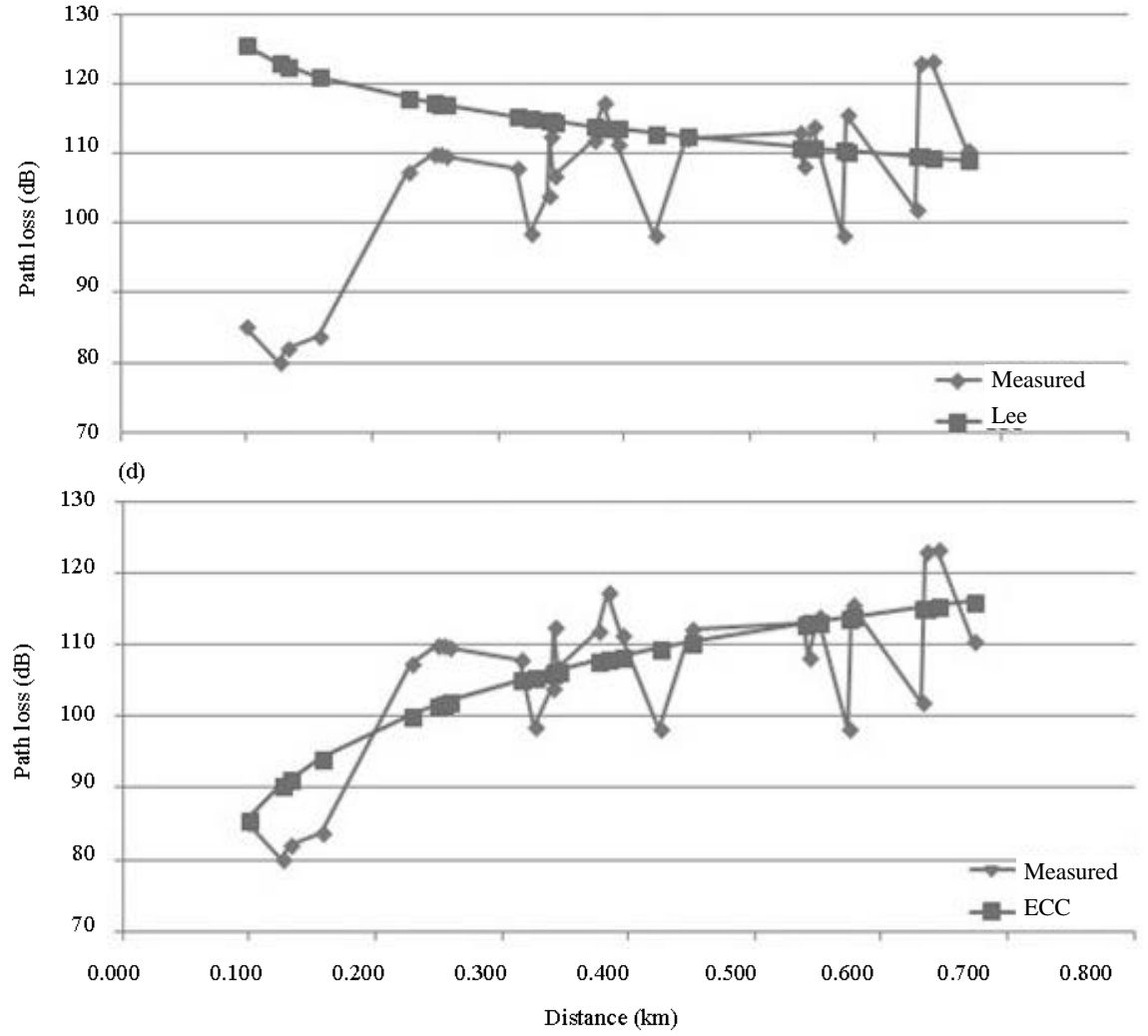

Fig. 4: a) Comparison of measured path loss with Hata Model; b) Comparison of measured path loss with Walfisch Ikegami Model; c) Comparison of measured path loss with Lee Model and d) Comparison of measured path loss with ECC Model 

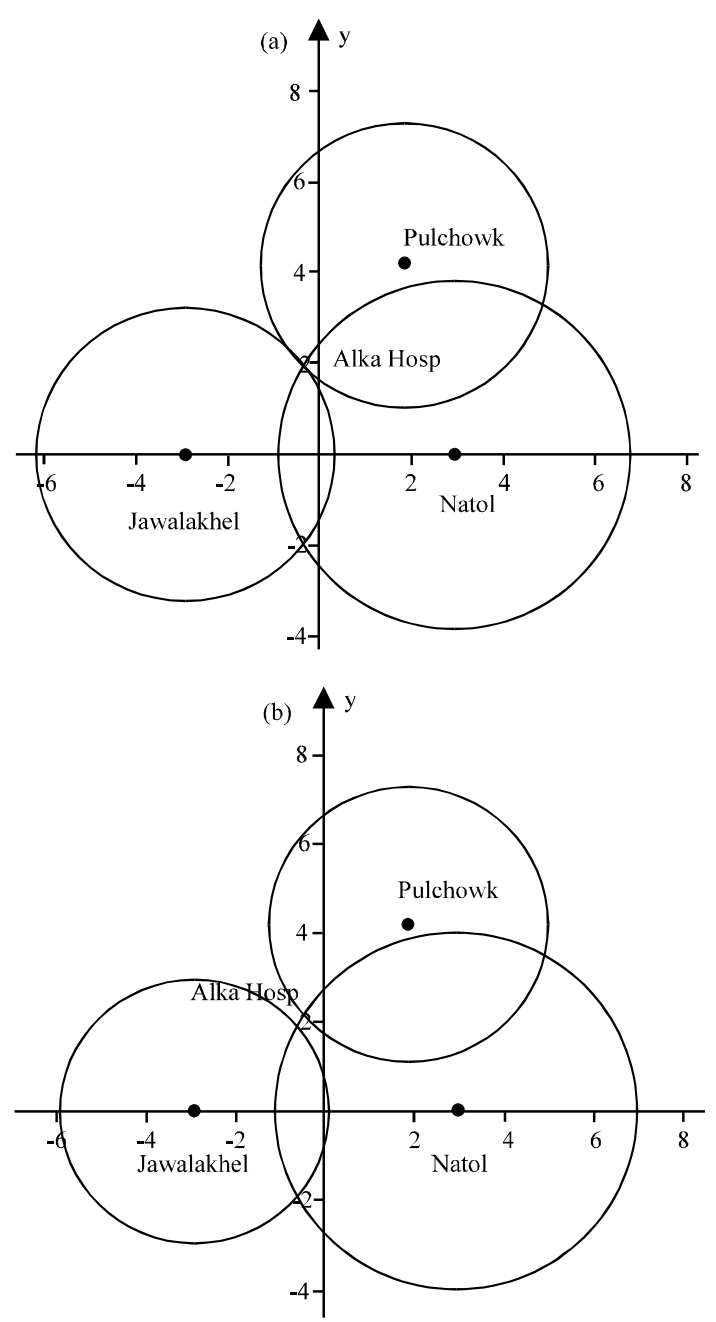

Fig. 5: a) Actual distance between BSs and MS and b) Estimated distance between BSs and MS

\section{CONCLUSION}

The mobile location is estimated using the RSS method. This method is capable of being applied in existing systems without any hardware development. The Walfisch Ikegami Model is used to calculate the distance between transmitter and receiver. The comparison is performed between the actual distance of the MS with the estimated distance using the Walfisch Ikegami Model. The accuracy obtained from WI Model was found to be $22 \mathrm{~m}$.

\section{REFERENCES}

Bhandare, S.S. and M. Dixit, 2013. Positioning of mobile in GSM network using received signal strength and angle of arrival. Intl. J. Emerg. Trend Technol. Comput. Sci., 2: 400-403.

Chebil, J., A.K. Lwas, M.R. Islam and A.H. Zyoud, 2011. Adjustment of Lee path loss model for suburban area in Kuala Lumpur-Malaysia. Proceedings of the International Conference on Computer Communication and Management (ICCCM 201 1), May 2-4, 201 1, International Islamic University Malaysia, Kuala Lumpur, Malaysia, pp: 252-257.

Iskander, M.F. and Z. Yun, 2002. Propagation prediction models for wireless communication systems. IEEE. Trans. Microwave Theory Tech., 50: 662-673.

Khan, I., T.C. Eng and S.A. Kamboh, 2012. Performance analysis of various path loss models for wireless network in different environments. Intl. J. Eng. Adv. Technol., 2: 161-165.

Lin, D.B. and R. T. Juang, 2005. Mobile location estimation based on differences of signal attenuations for GSM systems. IEEE Trans. Vehieular Technol., 54: 1447-1454.

Liu, H., H. Darabi, P. Banerjee and J. Liu, 2007. Survey of wireless indoor positioning techniques and systems. IEEE Trans. Syst. Man Cybern. Part C: Appl. Rev., 37: 1067-1080.

Parmar, K.J. and V.D. Nimavat, 2015. Comparative analysis of path loss propagation models in radio communication. Intl. J. Innovative Res. Comput. Commun. Eng., 3: 840-844.

Willmott, C.J. and K. Matsuura, 2005. Advantages of the mean absolute error (MAE) over the root mean square error (RMSE) in assessing average model performance. Climate Res., 30: 79-82. 